LA W RENCE LIVERMORE N A TIONAL LABORATORY
Final Report on Subcontract B601287: Auxiliary Space Preconditioners and Algebraic Multilevel Solvers for Systems of PDEs

J. Brannick

June 19, 2013 
This document was prepared as an account of work sponsored by an agency of the United States government. Neither the United States government nor Lawrence Livermore National Security, LLC, nor any of their employees makes any warranty, expressed or implied, or assumes any legal liability or responsibility for the accuracy, completeness, or usefulness of any information, apparatus, product, or process disclosed, or represents that its use would not infringe privately owned rights. Reference herein to any specific commercial product, process, or service by trade name, trademark, manufacturer, or otherwise does not necessarily constitute or imply its endorsement, recommendation, or favoring by the United States government or Lawrence Livermore National Security, LLC. The views and opinions of authors expressed herein do not necessarily state or reflect those of the United States government or Lawrence Livermore National Security, LLC, and shall not be used for advertising or product endorsement purposes.

This work performed under the auspices of the U.S. Department of Energy by Lawrence Livermore National Laboratory under Contract DE-AC52-07NA27344. 


\title{
Final Report on Subcontract B601287: Auxiliary Space Preconditioners and Algebraic Multilevel Solvers for Systems of PDEs
}

\author{
June 30, 2012 - May 31, 2013 \\ PI: Jinchao Xu, CoPI: James Brannick
}

Progress has been made in several of the proposed areas of study, as summarized below.

\section{Compatible Relaxation and Fourier Analysis}

The project team has continued with work on developing Compatible Relaxation algorithms for both Classical and Aggregation AMG. Of particular interest is the idea to use CR to adaptively construct the smoother, based on a new more sharp or predictive variant of compatible relaxation, several versions of habituated forms of CR have been developed and implemented, and in particular a block Gauss Seidel CR iteration was developed and analyzed for use with aggressive coarsening. The resulting smoothers and coarse space constructions have also been analyzed using local Fourier analysis (LFA) and shown to match with the predictive version of CR.

Improving aggregation-based MG solver performance -- Variable cycling.

We have developed a variable (algebraic multilevel iteration) cycle in the aggregationbased MG setting for the graph Laplace system. In this project period we designed and analyzed the method for use with aggressive coarsening and polynomial smoothers, and have proved the near optimality in the case of coarse spaces generated by graph partitioning. We have also confirmed the sharpness of the theoretical estimates numerically.

In terms of the polynomial smoother, we have analyzed the convergence properties of the method using LFA, considering several Chebychev-based polynomials, including a new polynomial approximation to the matrix inverse.

\section{Auxiliary Space Preconditioners for the Overlap Operator}

We conducted numerical tests that demonstrate that the low eigenvectors of the Wilson matrix are good approximations to the low eigenvectors of the Overlap Dirac matrix. We have implemented a defect-correction type method based on this idea and carried out extensive tests for the 2d Schwinger model of QED. In the free case, the method exhibits optimal performance, however, in the gauged case, it's performance is also nearly optimal for increasing problem size or varying masses, assuming that the Wilson matrix is nearly normal. Current efforts in this area focus on applying the defect correction scheme together with smearing techniques for the Wilson system. 
We developed a new adaptive Bootstrap AMG (BAMG) solver for the non-Hermitain Wilson Dirac system. The approach employs an adaptive setup algorithm to compute interpolation, based on the notion least squares fitting of test vectors, i.e, a set of vectors computed adaptively. This is an alternative, and potentially far less expensive approach to the adaptive aggregation-based setup algorithm we originally developed for the Wilson system.

The adaptive aggregation-based setup algorithm generates the prototypes used in defining Multigrid interpolation sequentially, which requires recomputing the MG hierarchy at each adaptive step. In contrast, our BAMG adaptive aggregation-based setup, computes interpolation in a single (or few) adaptive step(s) using as a main tool an adaptive multi-level eigensolver.

The new BAMG approach has been shown to yield a solver whose performance is better than that of the aggregation based scheme with less worked required in the adaptive setup.

\section{References}

1. J. Brannick and K. Kahl, Bootstrap AMG for the Wilson Dirac system, in preparation, 2013.

2. J. Brannick, Polynomial smoothing and aggressive aggregation-based coarsening, DD22 proceedings, submitted, 2012. 